\title{
Detectable and undetectable input signals for the integrate-and-fire model
}

\author{
Jianfeng Feng ${ }^{1,2}$, David Brown ${ }^{2}$, Gang Wei ${ }^{3}$ and Brunello Tirozzi ${ }^{4}$ \\ ${ }^{1}$ COGS, Sussex University, Brighton BN1 9QH, UK \\ 2 Computational Neuroscience Laboratory, The Babraham Institute, Cambridge CB2 4AT, UK \\ ${ }^{3}$ Department of Mathematics, Hong Kong Baptist University, Hong Kong, People's Republic of \\ China \\ ${ }^{4}$ Physics Department, Rome University 'La Sapienza', Rome 00185, Italy \\ E-mail: jf218@cam.ac.uk
}

Received 22 June 2000, in final form 8 December 2000

\begin{abstract}
We consider the integrate-and-fire model with non-stationary, stochastic inputs and address the following issue: what are the conditions on the input currents that make the input signal undetectable? A novel theoretical approach to tackle the problem for the model with non-stationary inputs is introduced. When the noise strength is independent of the deterministic component of the synaptic input, an expression for the critical input signal is given. If the input signal is weaker than the critical input signal, the neuron ultimately stops firing, i.e. is not able to detect the input signal; otherwise it fires with probability one. Similar results are established for Poisson type inputs where the strength of the noise is proportional to the deterministic component of the synaptic input.
\end{abstract}

PACS numbers: 0540J, 0250, 0590, 8435, 8719L

\section{Introduction}

Single neuron models with stochastic inputs have been extensively studied during the past decades $[1,4,6,7,13,18,23-25,27,28]$. Much as most theoretical studies focus on models with constant or stationary inputs ${ }^{5}$, it is obvious that models with non-stationary inputs are of primary interest. In fact, there is growing literature devoted to the study of biological neurons with natural scene inputs; see, e.g., [22].

Here we consider the simplest neuronal model, the integrate-and-fire model with nonstationary inputs. The question to be addressed is: which kind of change in the input signal is a neuron not capable of detecting? In other words, what is the neuron's detection limit in terms of input signals? By detection of an input signal for a neuron, we mean that the neuron fires spikes if the signal is presented. At a first glance, we might think that it is totally impossible to

5 Models studied in stochastic resonance are exceptional, where periodic or aperiodic input signals are considered [5, $15,17]$. 
shut down a neuron's response to stochastic inputs. This is true for stationary inputs: a neuron will fire with probability one due to noise. The situation is totally different for non-stationary inputs.

Here, by employing a theoretical method called the Poisson clumping heuristic, developed in [3], we establish two fundamental theorems on neuronal responses to input signals (see sections 3 and 4 for exact formulations).

Case 1. When the noise amplitude is independent of the deterministic component of the synaptic input, as for many models considered in stochastic resonance, a critical phenomenon is found. More specifically, let the synaptic input $I(t)$ of the integrate-and-fire model be

$$
\mathrm{d} I(t)=\mu \lambda(t) \mathrm{d} t+\sigma \mathrm{d} B_{t}
$$

where $B_{t}$ is the standard Brownian motion, $t$ is the time, $\sigma$ the noise strength and $\mu \lambda(t)$ the input signal. If, for large $t$, the input signal decreases faster than $-\sigma \sqrt{2 / \gamma} \sqrt{\log (t / \gamma+C)}$, a neuron is ultimately not able to respond to it; otherwise the neuron fires with probability one, where $\gamma$ is the decay time of the integrate-and-fire model and $C>0$ a constant. For example, when $\mu \lambda(t)=-t$, the neuron is ultimately not able to respond to it.

Case 2. For Poisson type inputs, i.e.

$$
\mathrm{d} I(t)=\mu \lambda(t) \mathrm{d} t+\sigma \sqrt{\lambda(t)} \mathrm{d} B_{t}
$$

if $\mu \lambda(t)$ decreases faster than $-\sigma \sqrt{2 / \gamma} \log (t / \gamma+C)$, a neuron is not able to respond to it; otherwise the neuron fires with probability one.

As might be expected, Poisson type inputs improve the detectable limit of a neuron. For example, for Poisson type inputs with $\mu \lambda(t)=-2 \sigma \sqrt{2 / \gamma} \sqrt{\log (t / \gamma+C)}$, the integrate-andfire neuron will fire with probability one, but in the circumstances of case 1 the neuron will be ultimately silent.

A central issue in neuroscience is the coding problem $[2,16,26]$. The way a neuron encodes and then decodes inputs is still a mystery, despite a century of research. To understand the detectable limit of a neuron will certainly help us gain some more insights into the coding problem. Furthermore we expect that techniques developed here will also be valuable for studying other issues for neuronal models with non-stationary inputs. The integrate-and-fire model is the simplest and the most widely used neuronal model. We have learnt a lot from studying it alone, as has been amply demonstrated in the literature. The results presented here are, rigorously speaking, only proved for the integrate-and-fire model. It is expected, however, that the conclusions drawn here may be enlightening when more complex, biophysical neuronal models are considered.

\section{The model}

Suppose that a cell receives EPSPs (excitatory postsynaptic potentials) at $N_{\mathrm{E}}$ excitatory synapses and IPSPs (inhibitory postsynaptic potentials) at $N_{\mathrm{I}}$ inhibitory synapses. The activities among excitatory synapses and inhibitory synapses are correlated with correlation coefficient $c[13,19,29]$, and are assumed to be independent between them (see the discussion section and [13]). When the membrane potential $Z_{t}$ is between the resting potential $V_{\text {rest }}$ and the threshold $V_{\text {thre }}$, its time evolution is given by

$$
\mathrm{d} Z_{t}=-\frac{1}{\gamma}\left(Z_{t}-V_{\text {rest }}\right) \mathrm{d} t+a \sum_{i=1}^{N_{\mathrm{E}}} \mathrm{d} E_{i}(t)-b \sum_{j=1}^{N_{\mathrm{I}}} \mathrm{d} I_{j}(t)
$$


where $1 / \gamma$ is the decay rate, $E_{i}(t), I_{i}(t)$ are Poisson processes with rates $\lambda_{\mathrm{E}}(t)$ and $\lambda_{\mathrm{I}}(t)$, respectively, and $a, b$ are magnitudes of each EPSP and IPSP $[4,8,9]$. Once $Z_{t}$ crosses $V_{\text {thre }}$ from below a spike is generated and $Z_{t}$ is reset to $V_{\text {rest }}$. This model is termed the integrateand-fire model. We further assume that $\lambda_{\mathrm{E}}(t)=\lambda_{\mathrm{I}}(t)=\lambda(t)$ (see the discussion section). The integrate-and-fire model with a Poisson process input is the most commonly studied case in the literature. Certainly it is an over-simplification of the actual situation [18] and a discussion of it is outside the scope of this paper. Furthermore we have not included reversal potentials here, which will cause more difficulties in analysing the model.

From now on we assume that $V_{\text {rest }}=0 \mathrm{mV}$. In fact, we are not going to deal with the discrete processes $Z_{t}$; instead we consider the continuous approximation version of it given by $[8-12,20,21,28]$

$$
\mathrm{d} V_{t}=-\frac{1}{\gamma} V_{t} \mathrm{~d} t+\mathrm{d} I(t)
$$

where the synaptic input $\mathrm{d} I(t)=\mu(t) \mathrm{d} t+\sigma(t) \mathrm{d} B_{t}$ with

$$
\begin{aligned}
& \mu(t)=\left(a N_{\mathrm{E}}-b N_{\mathrm{I}}\right) \lambda(t)=\mu \lambda(t)(\text { drift term }) \\
& \sigma^{2}(t)=\left[a^{2} N_{\mathrm{E}}+b^{2} N_{\mathrm{I}}+a^{2} c\left(N_{\mathrm{E}}^{2}-N_{\mathrm{E}}\right)+b^{2} c\left(N_{\mathrm{I}}^{2}-N_{\mathrm{I}}\right)\right] \lambda(t)=\sigma^{2} \lambda(t)
\end{aligned}
$$

and $B_{t}$ is the standard Brownian motion. The interspike interval of efferent spikes (the firing time) is

$$
T=\inf \left\{t: V_{t} \geqslant V_{\text {thre }}\right\}
$$

\section{Constant diffusion coefficient}

We first consider a simple case with the diffusion coefficient independent of time $(\sigma(t)=\sigma)$ :

$$
\mathrm{d} V_{t}=-\frac{V_{t}}{\gamma} \mathrm{d} t+\mu \lambda(t) \mathrm{d} t+\sigma \mathrm{d} B_{t}
$$

This type of model is widely used in stochastic resonance and we refer the reader to, for example [17], for further discussion.

We rewrite the equation above into a canonical form by introducing a transformation [14] $s=t / \gamma$ and $X_{s}=V_{\gamma s}=V_{t}$ :

$$
\mathrm{d} X_{s}=-X_{s} \mathrm{~d} s+\mu \gamma \lambda(\gamma s) \mathrm{d} s+\sigma \sqrt{\gamma} \frac{\mathrm{d} B_{\gamma s}}{\sqrt{\gamma}} .
$$

Since $\mathrm{d} \bar{B}_{s}=\mathrm{d} B_{\gamma_{s}} / \sqrt{\gamma}$ is again a standard Brownian motion, equation (3.2) becomes

$$
\mathrm{d} X_{s}=-X_{s} \mathrm{~d} s+\mu \gamma \lambda(\gamma s) \mathrm{d} s+\sigma \sqrt{\gamma} \mathrm{d} \bar{B}_{s} .
$$

The first-exit time (firing time) is then

$$
\begin{aligned}
T & =\inf \left\{t: V_{t} \geqslant V_{\text {thre }}\right\} \\
& =\gamma \inf \left\{s: X_{s} \geqslant V_{\text {thre }}\right\} .
\end{aligned}
$$

Solving equation (3.3) we obtain

$$
X_{u}=\gamma \mu \int_{0}^{u} \exp [-(u-s)] \lambda(\gamma s) \mathrm{d} s+\sigma \sqrt{\gamma} \int_{0}^{u} \exp [-(u-s)] \mathrm{d} \bar{B}_{s} .
$$


Hence

$T=\inf \left\{u: \int_{0}^{u} \exp [-(u-s)] \mathrm{d} \bar{B}_{s} \geqslant \frac{1}{\sigma \sqrt{\gamma}}\left\{V_{\text {thre }}-\mu \gamma \int_{0}^{u} \exp [-(u-s)] \lambda(\gamma s) \mathrm{d} s\right\}\right\}$.

Note that $\int_{0}^{u} \exp [-(u-s)] \mathrm{d} \bar{B}_{s}$ is a standard Ornstein-Uhlenbeck process. From the results of Poisson clumping heuristic (equation (D15a) on p 85 of [3]), we know that for $t \geqslant 0$

$$
P(T \geqslant t)=1-\exp \left[-\int_{0}^{t} b(u) \cdot \exp \left\{-b^{2}(u) / 2\right\} \mathrm{d} u\right]
$$

where

$$
b(u)=\frac{V_{\text {thre }}-\mu \gamma \int_{0}^{u} \exp [-(u-s)] \lambda(\gamma s) \mathrm{d} s}{\sqrt{\gamma} \sigma} \geqslant 0 .
$$

Note that the requirement of $b(u)$ being non-negative implies that stimuli are subthreshold. For suprathreshold stimuli we certainly have $P(T<\infty)=1$ (see theorem 1 below). Equation (3.7) gives rise to an informative description of the input-output relationship of the integrate-and-fire model with arbitrary (subthreshold) inputs. For example, the distribution density $p(t)$ of $T$ (the derivative of $P(T \geqslant t)$, equation (3.7)) is given by

$$
p(t)=b(t) \exp \left(-b^{2}(t) / 2\right) \exp \left[-\int_{0}^{t} b(u) \exp \left(-b^{2}(u) / 2\right) \mathrm{d} u\right]
$$

Figure 1 depicts the distribution density of $T$ versus time, in the case when the input signal is strictly periodic, i.e. $\lambda(t)=a(\cos (\omega t)+b)$ where $a, b, \omega$ are all constants. It is very interesting to see how the output histogram is affected by the input periodic signal. When $\omega$ is large ( $\omega=1$ in figure 1), the histogram oscillates locally. When $\omega$ becomes small (see figure 1 with $\omega=0.1$ and 0.01 ), the oscillation disappears, as expected.

Next we determine the detectable input signal for the model we consider. To this end, we suppose that

$$
\lambda(\gamma s)=\frac{V_{\text {thre }}}{\mu \gamma}+\exp (-\gamma s / \gamma) a(\gamma s)
$$

for a function $a$ which will be specified below. $b(u)$ defined by equation (3.8) turns out to be (all higher order terms in the equality are omitted)

$$
b(u)=-\frac{\mu \sqrt{\gamma} \int_{0}^{u} \exp [-u] a(\gamma s) \mathrm{d} s}{\sigma} .
$$

Substituting the expression of $b(u)$ above into equation (3.7), we have

$$
\begin{aligned}
P(T=\infty)= & 1-\exp \left[\int_{0}^{\infty}\left\{\frac{\mu \sqrt{\gamma} \int_{0}^{u} \exp (-u) a(\gamma s) \mathrm{d} s}{\sigma}\right\}\right. \\
& \left.\times \exp \left\{-\frac{1}{2}\left(\frac{\mu \sqrt{\gamma} \int_{0}^{u} \exp (-u) a(\gamma s) \mathrm{d} s}{\sigma}\right)^{2}\right\} \mathrm{d} u\right] .
\end{aligned}
$$

Therefore, if $P(T \geqslant t)$ is to approach unity as $t \rightarrow \infty$, then it is equivalent to require that the following quantity:

$$
\int_{0}^{\infty}\left[\int_{0}^{u} \exp (-u) a(r s) \mathrm{d} s\right] \exp \left[-\frac{\left(\mu \exp (-u) \int_{0}^{u} a(r s) \mathrm{d} s\right)^{2} \gamma}{2 \sigma^{2}}\right] \mathrm{d} u
$$

should diverge. We then see that $\gamma \mu^{2}=2 \sigma^{2}$ and $a(\gamma s)=\exp (\gamma s / \gamma) \sqrt{\log (\gamma s / \gamma+C)}$ is the critical case. 

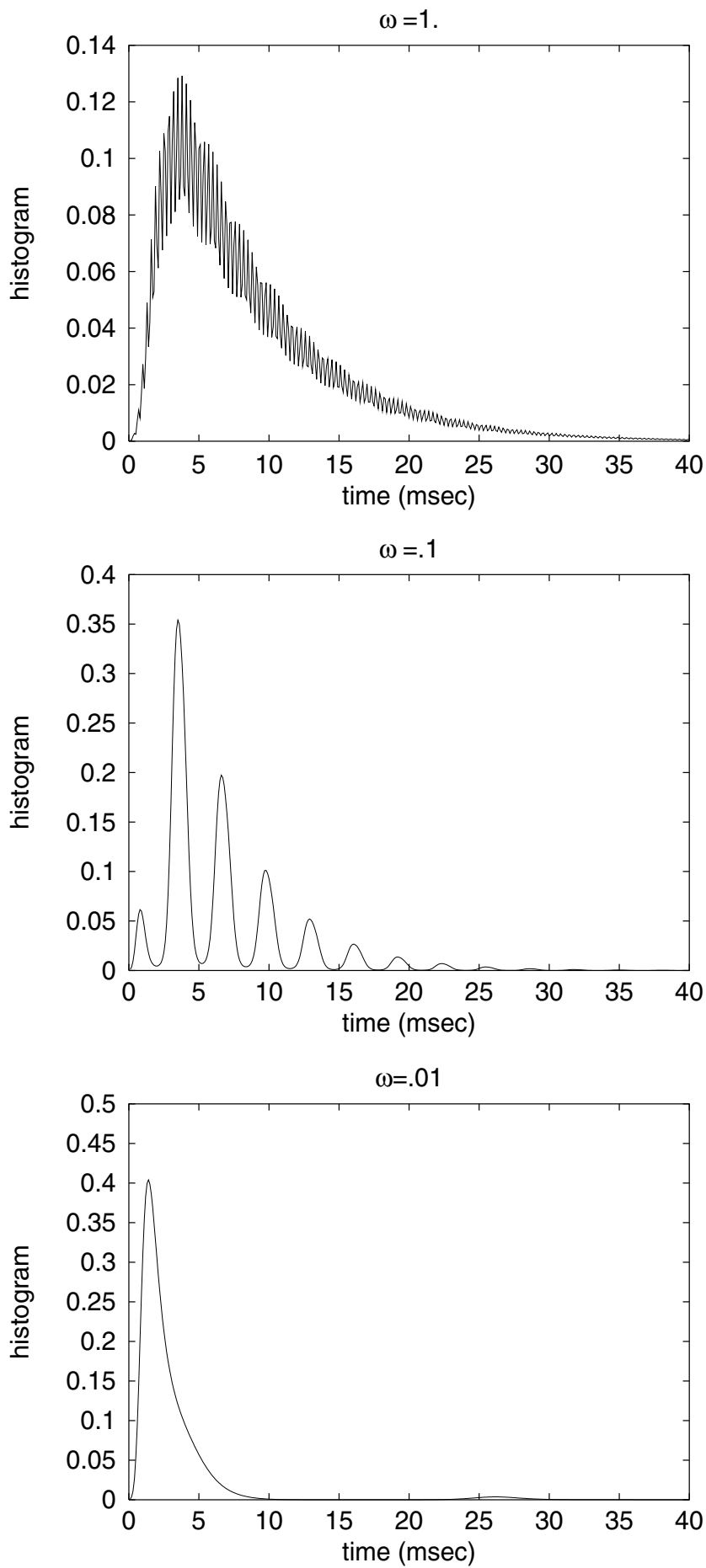

Figure 1. Distribution density (histogram) of $T$ versus time with $\lambda(t)=0.5(\cos (\omega t)+1), \sigma=1$, $\gamma=20 \mathrm{~ms}, V_{\text {thre }}=20 \mathrm{mV}$ and $\mu=1$, constant diffusion coefficient. 


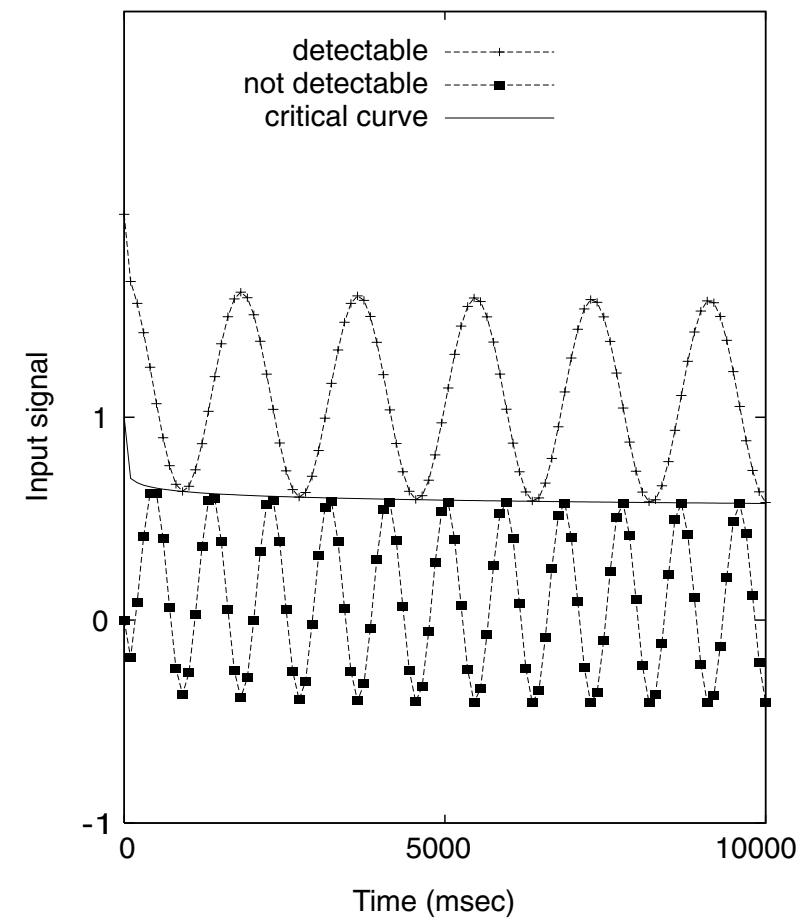

Figure 2. Input signal $\mu \lambda(t)$ versus time. Critical curves $=\mu \lambda(t)$ with $\mu=-\sqrt{2}, \sigma=\sqrt{\gamma}$, $C=10, V_{\text {thre }}=20 \mathrm{mV}$ and $\gamma=20 \mathrm{~ms}$.

In conclusion, considering the critical value for the input rate

then:

$$
\lambda(t)=\frac{V_{\text {thre }}}{\mu \gamma}+\sqrt{\log (t / \gamma+C)}
$$

- if $\mu<-\sigma \sqrt{2 / \gamma}$ and $C$ is large enough, then the signal is undetectable, that is to say $P(T=\infty)=1$,

- if $\mu>-\sigma \sqrt{2 / \gamma}$ and $C>0$, then the signal is detectable, that is to say $P(T<\infty)=1$.

For the general case of $\lambda(t)$, by combining the arguments above, we have the following theorem.

Theorem 1 (Detectable theorem I). We consider the integrate-and-fire model defined by equation (3.1) (see figure 2).

- If

$$
\mu \cdot \limsup _{t \rightarrow \infty} \frac{\lambda(t)}{\sqrt{\log \left(\frac{t}{\gamma}+C\right)}}<-\sigma \sqrt{\frac{2}{\gamma}}
$$

and $C$ is large enough, the input signal is undetectable, i.e. $P(T=\infty)=1$.

- If

$$
\mu \cdot \liminf _{t \rightarrow \infty} \frac{\lambda(t)}{\sqrt{\log \left(\frac{t}{\gamma}+C\right)}}>-\sigma \sqrt{\frac{2}{\gamma}}
$$

and $C>0$, the signal is detectable, i.e. $P(T<\infty)=1$. 
Theorem 1 states that for the critical situation, i.e. $\lambda(t) \sim \sqrt{\log (t / \gamma+C)}$, if the inhibition is strong enough $a N_{\mathrm{E}}-b N_{\mathrm{I}}<-\sigma \sqrt{2 / \gamma}$ (see equation (2.3) and below), then the neuron ultimately stop firing, otherwise it fires with a probability of one. For the general case when the drift term is smaller than $-\sigma \sqrt{2 / \gamma} \sqrt{\log (t / \gamma+C)}$, the neuron ultimately stop firing; otherwise it fires with a probability of one.

The following example gives a concrete example to explain the results of theorem 1.

Example 1. Suppose that $\lambda(t)=t+C$ and $\mu=-1, \sigma=1$ and $\gamma=1$. From equation (3.7) we conclude that

$$
P(T=\infty)=1-\sqrt{\frac{V_{\text {thre }}}{2 C}} \exp \left(-V_{\text {thre }}^{2} / 2\right)
$$

which tends to 1 as $C$ tends to infinity.

Let us have another look at the exact meaning of detectable and undetectable input signals. For a fixed, detectable input as depicted in figure 2, the neuron fires with probability one, starting at any time. In other words, $P\left(t_{0}<T<\infty\right)=1$. In contrast, when the input is undetectable, the probability of firing vanishes as time goes on, i.e. $P\left(t_{0}<T<\infty\right) \rightarrow 0$ with $t_{0} \rightarrow \infty$.

\section{Poisson type inputs}

We consider a neuronal model of the following form, i.e. a diffusion approximation to a Poisson input:

$$
\mathrm{d} V_{t}=-\frac{V_{t}}{\gamma} \mathrm{d} t+\mu \lambda(t) \mathrm{d} t+\sigma \sqrt{\lambda(t)} \mathrm{d} B_{t}
$$

where $\mu, \sigma$ are both constants. Again from the Poisson clumping heuristic (D18 on p 87 of [3]), we conclude that the distribution density $p(t)$ of $T$ is given by (see figure 3 )

$$
p(t)=\bar{b}(t) \exp \left(-\bar{b}^{2}(t) / 2\right) \exp \left[-\int_{0}^{t} \bar{b}(u) \exp \left(-\bar{b}^{2}(u) / 2\right) \mathrm{d} u\right]
$$

where

$$
\bar{b}(u)=\frac{V_{\text {thre }}-\mu \int_{0}^{u} \exp [-(u-s)] \gamma \lambda(\gamma s) \mathrm{d} s}{\sqrt{\int_{0}^{u} \exp [-2(u-s)] \gamma \lambda(\gamma s) \sigma^{2} \mathrm{~d} s}} .
$$

A comparison of figure 1 with 3 reveals the difference between inputs of constant diffusion coefficient and of Poisson type (a more biologically plausible case). Nevertheless, in terms of distribution densities, the difference is obvious only when input signals oscillate fast (large $\omega)$. In fact, it would be more informative to compare the function $b(u)$ with $\bar{b}(u)$ since, in the expression for the distribution densities, the temporal information of $b(u)$ or $\bar{b}(u)$ is averaged out. We call $b(u)$ and $\bar{b}(u)$ response functions. The justification lies in the fact that, for example, in equation (4.2), when $\bar{b}$ is small and independent of time it is simply the firing rate of the efferent spike trains. Figure 4 shows that there is a sharp difference between the response functions $b(u)$ and $\bar{b}(u)$ even when $\omega$ is small. For the input with a constant coefficient, the response function is similar to the input; while for the input of Poisson type, the response function is more concentrated on the peaks of the input signal (with a phase lag). This phenomenon is very interesting and we are going to explore it further in another publication.

Finally from equation (4.2) we know that $P(T=\infty)=1$ if and only if $\int_{0}^{\infty} \bar{b}(u) \exp \left\{-\bar{b}^{2}(u) / 2\right\} \mathrm{d} u$ is finite.

After repeating similar arguments as in the previous sections we reach the following conclusions. 

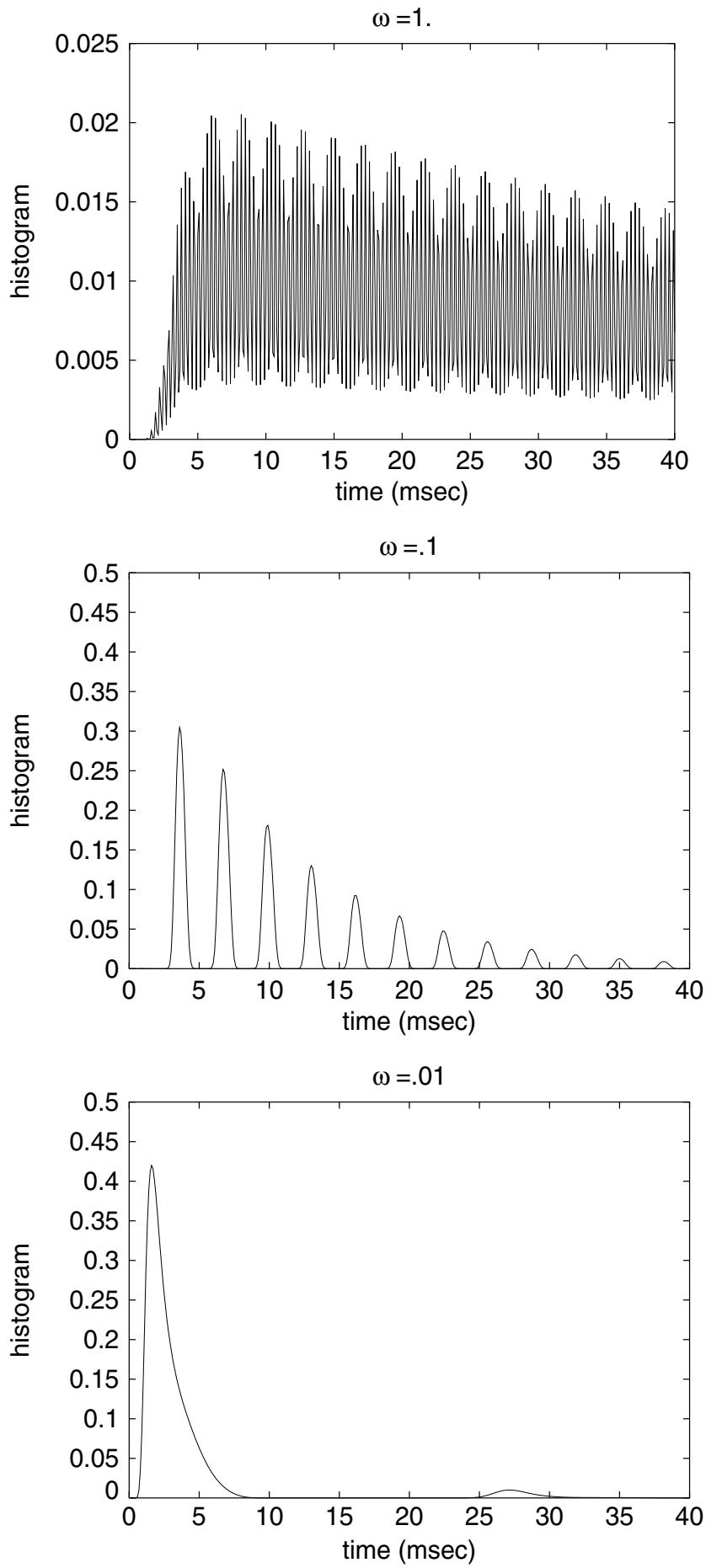

Figure 3. Histograms versus time with $\lambda(t)=0.45(\cos (\omega t)+1), \sigma=1, \gamma=20 \mathrm{~ms}$, $V_{\text {thre }}=20 \mathrm{mV}$ and $\mu=1$, Poisson type inputs. 


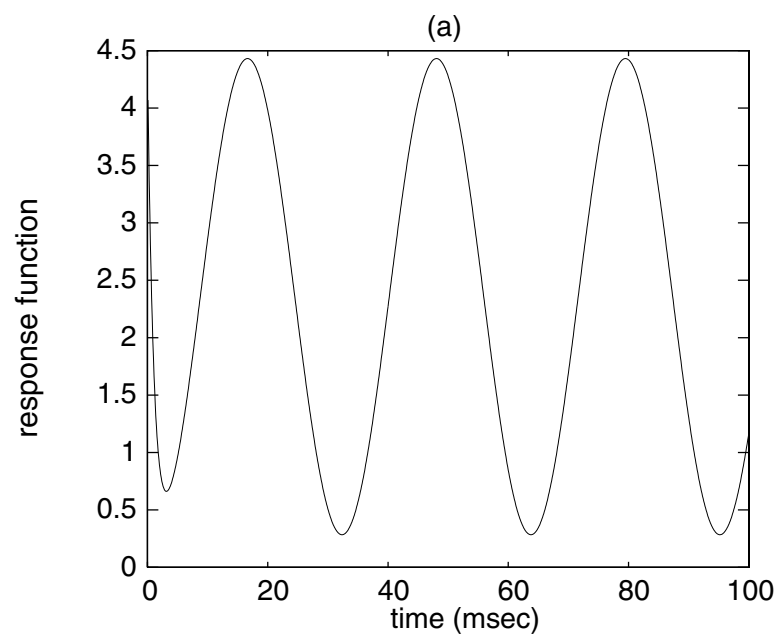

(b)

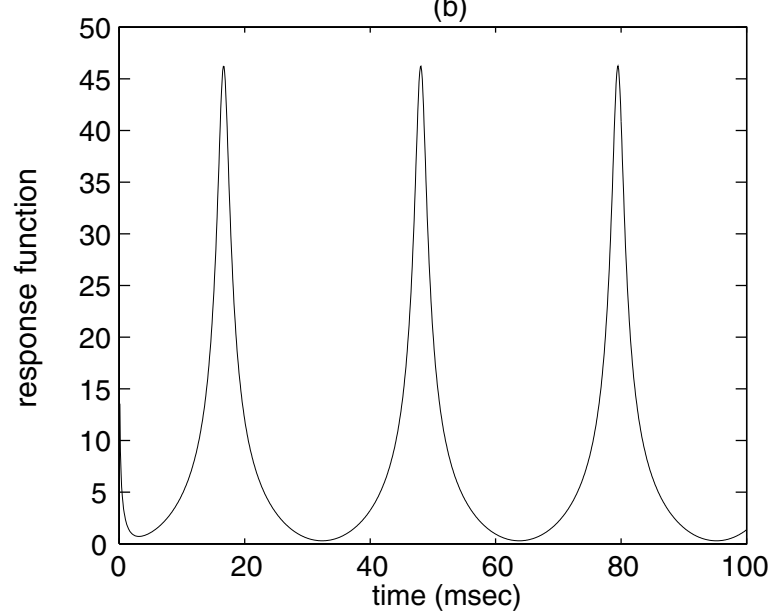

(c)

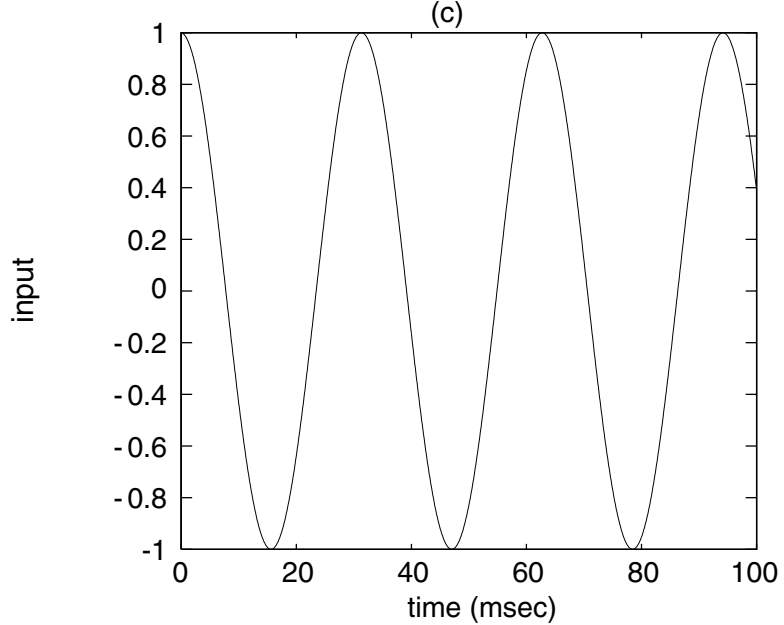

Figure 4. (a) $b(t)$ defined by equation (3.8) versus $s$, (b) $\bar{b}(t)$ defined by equation (4.3) versus $s$ and $(c)$ input $(\cos (\omega t))$ versus time. $\lambda(t)=0.45(\cos (\omega t)+1), \sigma=1, \gamma=20 \mathrm{~ms}, \omega=0.01$, $V_{\text {thre }}=20 \mathrm{mV}$ and $\mu=1$. 
Theorem 2 (Detectable theorem II). For the integrate-and-fire model defined by equation (4.1) the following is true.

- If

$$
\mu \cdot \limsup _{t \rightarrow \infty} \frac{\lambda(t)}{\log \left(\frac{t}{\gamma}+C\right)}<-\sigma \sqrt{\frac{2}{\gamma}}
$$

and $C$ is large enough, the input signal is undetectable, i.e. $P(T=\infty)=1$.

- If

$$
\mu \cdot \liminf _{t \rightarrow \infty} \frac{\lambda(t)}{\log \left(\frac{t}{\gamma}+C\right)}>-\sigma \sqrt{\frac{2}{\gamma}}
$$

and $C>0$, the input signal is detectable, i.e. $P(T<\infty)=1$.

Theorem 2 states that with a Poisson type input, the detectability of the input signal by the model neuron is improved since $P(T<\infty)=1$ even when $\mu \lambda(t)>-\sqrt{2 / \gamma} \sigma \log (t / \gamma+C)$, in comparison with the case of a constant diffusion coefficient where $P(T=\infty)=1$ even when $\mu \lambda(t)<-\sqrt{2 / \gamma} \sigma \sqrt{\log (t / \gamma+C)}$.

\section{How to shut down response at the critical case}

In order to fully understand our results, we further analyse the critical case when $\lambda(t)=$ $\sqrt{\log (t / \gamma+C)}$ (for the constant diffusion coefficient case) or $\lambda(t)=\log (t / \gamma+C$ ) (for the Poisson type input case), i.e. we want to know how a neuron could shut down its response to input signals. Let us introduce some more notation. Denote $a=b, N=N_{\mathrm{E}}, r=N_{\mathrm{I}} / N_{\mathrm{E}}$ and suppose that $c=0$. From equation (2.3) we see that $\gamma \mu^{2}=2 \sigma^{2}$ is equivalent to $\gamma N(1-r)^{2}=2(1+r)$. Therefore when $N \gamma$ is large (which is the case: see, e.g., [7-13]), $r \sim 1$. This means that the critical case occurs when inhibitory inputs and excitatory inputs are exactly balanced [24,25]. Hence when the inhibitory input is stronger than the excitatory input, the neuron ultimately stops firing; otherwise it fires with probability one.

\section{Discussion}

In summary we have established two fundamental theorems on which kind of input signal is detectable by the integrate-and-fire model. We hope the theoretical approach introduced here will be widely applicable to issues related to the integrate-and-fire model with non-stationary inputs.

Finally we return to the assumptions in section 2.

- Correlation between excitatory and inhibitory inputs. Suppose that the correlation coefficient between excitatory and inhibitory inputs is $c^{\mathrm{I}, \mathrm{E}}$, i.e. we relax the assumption that the excitatory and inhibitory inputs are independent of each other, then $\sigma^{2}(t)$ in equation (2.3) turns out to be

$$
\sigma^{2}(t)=\left[a^{2} N_{\mathrm{E}}+b^{2} N_{\mathrm{I}}+a^{2} c\left(N_{\mathrm{E}}^{2}-N_{\mathrm{E}}\right)+b^{2} c\left(N_{\mathrm{I}}^{2}-N_{\mathrm{I}}\right)-2 a b N_{\mathrm{E}} N_{\mathrm{I}} c^{I, E}\right] \lambda(t)=\sigma^{2} \lambda(t) .
$$

All results developed in the previous sections are true mutatis mutandis. 
- The assumption that $\lambda_{\mathrm{E}}(t)=\lambda_{\mathrm{I}}(t)=\lambda(t)$. In general we have (see equation $\left.(6.1)\right)$

$$
\begin{aligned}
& \mu(t)=a N_{\mathrm{E}} \lambda_{\mathrm{E}}(t)-b N_{\mathrm{I}} \lambda_{\mathrm{I}}(t)(\text { drift term }) \\
& \sigma^{2}(t)=a^{2} N_{\mathrm{E}} \lambda_{\mathrm{E}}(t)+b^{2} N_{\mathrm{I}} \lambda_{\mathrm{I}}(t)+a^{2} c\left(N_{\mathrm{E}}^{2}-N_{\mathrm{E}}\right) \lambda_{\mathrm{E}}(t) \\
& +b^{2} c\left(N_{\mathrm{I}}^{2}-N_{\mathrm{I}}\right) \lambda_{\mathrm{I}}(t)-2 a b N_{\mathrm{E}} N_{\mathrm{I}} c^{I, E} \sqrt{\lambda_{\mathrm{E}}(t) \lambda_{\mathrm{I}}(t)} .
\end{aligned}
$$

In principle, our theory developed in the previous sections is applicable, but would not be as clear-cut as theorems 1 and 2.

\section{Acknowledgments}

We would like to thank Peter Williams and an anonymous referee for their valuable comments on the paper. The paper was partially supported by BBSRC and an ESEP grant of the Royal Society.

\section{References}

[1] Abbott L F, Varela J A, Sen K and Nelson S B 1997 Synaptic depression and cortical gain control Science 275 220-3

[2] Albright T D, Jessell T M, Kandel E R and Posner M I 2000 Neural science: a century of progress and the mysteries that remain Cell 100 s $1-55$

[3] Aldous D 1989 Probability Approximation Via The Poisson Clumping Heuristic (Berlin: Springer)

[4] Brown D, Feng J and Feerick S 1999 Variability of firing of Hodgkin-Huxley and FitzHugh-Nagumo neurons with stochastic synaptic input Phys. Rev. Lett. 82 4731-4

[5] Collins J J, Chow C C and Imhoff T T 1995 Stochastic resonance without tuning Nature 376 236-8

[6] Destexhe A and Par D 1999 Impact of network activity on the integrative properties of neocortical pyramidal neurons in vivo J. Neurophysio. 81 1531-47

[7] Feng J 1997 Behaviours of spike output jitter in the integrate-and-fire model Phys. Rev. Lett. 79 4505-8

[8] Feng J and Brown D 1998a Spike output jitter, mean firing time and coefficient of variation J. Phys. A: Math. Gen. 31 1239-52

[9] Feng J and Brown D 1998b Impact of temporal variation and the balance between excitation and inhibition on the output of the perfect integrate-and-fire model Biol. Cybern. 78 369-76

[10] Feng J and Brown D 1999 Coefficient of variation greater than .5 How And When? Biol. Cybern. 80 291-7

[11] Feng J and Brown D 2000a Integrate-and-fire models with nonlinear leakage Bull. Math. Biol. 62 467-81

[12] Feng J, Brown D and Li G 2000 Synchronization due to common pulsed input in Stein's model Phys. Rev. E 61 2987-95

[13] Feng J and Brown D 2000b Impact of correlated inputs on the output of the integrate-and-fire models Neural Comput. 12 711-32

[14] Feng J, Georgii H O and Brown D 2000 Convergence to global minima for a class of diffusion processes Physica A $276465-76$

[15] Feng J and Tirozzi B 2000 Stochastic resonance tuned by correlations in neuronal models Phys. Rev. E 61 4207-11

[16] Gerstner W, Kreiter A K, Markram H and Herz A V M 1997 Neural codes: firing rates and beyond Proc. Natl. Acad. Sci. USA 94 12740-1

[17] Gammaitoni L, Hänggi P, Jung P and Marchesoni F 1998 Stochastic resonance Rev. Mod. Phys. 70 224-87

[18] Koch C 1997 Computation and the single neuron Nature 385 207-10

[19] Mattews P B C 1996 Relationship of firing intervals of human motor units to the trajectory of post-spike afterhyperpolarization and synaptic noise $J$. Physiol. 492 597-628

[20] Musila M and Lánský P 1994 On the interspike intervals calculated from diffusion approximations for Stein's neuronal model with reversal potentials J. Theor. Biol. 171 225-32

[21] Ricciardi L M and Sato S 1990 Diffusion process and first-passage-times problems Lectures in Applied Mathematics and Informatics ed L M Ricciardi (Manchester: Manchester University Press)

[22] Stanley G B, Li F F and Dan Y 1999 Reconstruction of natural scenes from ensemble responses in the lateral geniculate nucleus J. Neurosci. 19 8036-42

[23] Softky W and Koch C 1993 The highly irregular firing of cortical-cells is inconsistent with temporal integration of random EPSPs J. Neurosci. 13 334-50 
[24] Shadlen M N and Newsome W T 1994 Noise, neural codes and cortical organization Curr. Opin. Neurobiol. 4 $569-79$

[25] Shadlen M N and Newsome W T 1998 The variable discharge of cortical neurons: implications for connectivity, computation, and information coding J. Neurosci. 18 3870-96

[26] Shadlen MN and Movshon J A 1999 Synchrony unbound: a critical evaluation of the temporal binding hypothesis Neuron 24 67-77

[27] Stevens C F and Zador A M 1998 Input synchrony and the irregular firing of cortical neurons Nature Neurosci. $1210-7$

[28] Tuckwell H C 1988 Stochastic Processes in the Neurosciences (Philadelphia, PA: Society for Industrial and Applied Mathematics)

[29] Zohary E, Shadlen M N and Newsome W T 1994 Correlated neuronal discharge rate and its implications for psychophysical performance Nature 370 140-3 